\title{
Ubiquitination and SUMOylation in Telomere Maintenance and Dysfunction
}

\author{
Zeliha Yalçin, Carolin Selenz and Jacqueline J. L. Jacobs* \\ Department of Molecular Oncology, Netherlands Cancer Institute, Amsterdam, Netherlands
}

Telomeres are essential nucleoprotein structures at linear chromosomes that maintain genome integrity by protecting chromosome ends from being recognized and processed as damaged DNA. In addition, they limit the cell's proliferative capacity, as progressive loss of telomeric DNA during successive rounds of cell division eventually causes a state of telomere dysfunction that prevents further cell division. When telomeres become critically short, the cell elicits a DNA damage response resulting in senescence, apoptosis or genomic instability, thereby impacting on aging and tumorigenesis. Over the past years substantial progress has been made in understanding the role of post-translational modifications in telomere-related processes, including telomere maintenance, replication and dysfunction. This review will focus on recent findings that establish an essential role for ubiquitination and SUMOylation at telomeres.

\section{OPEN ACCESS}

Edited by:

Kristijan Ramadan,

University of Oxford, United Kingdom

Reviewed by:

Anabelle Decottignies,

Université catholique de Louvain,

Belgium

Howard Donninger,

University of Louisville, United States

*Correspondence:

Jacqueline J. L. Jacobs j.jacobs@nki.nl

Specialty section:

This article was submitted to Cancer Genetics,

a section of the journal

Frontiers in Genetics

Received: 16 December 2016

Accepted: 10 May 2017

Published: 23 May 2017

Citation:

Yalçin Z, Selenz C and Jacobs JJL

(2017) Ubiquitination and SUMOylation in Telomere Maintenance and Dysfunction.

Front. Genet. 8:67.

doi: 10.3389/fgene.2017.00067
Keywords: ubiquitin, SUMO, telomere maintenance, telomere dysfunction, DNA damage, DNA repair, shelterin, telomerase

\section{INTRODUCTION}

Genome stability is essential for cells to function properly and ensure the survival of an organism. At the ends of chromosomes this stability is maintained by telomeres. In vertebrates telomeres consist of long double-stranded stretches of TTAGGG repeats, ending in a 50-500 base pair overhang of the G-rich $3^{\prime}$-strand (Palm and de Lange, 2008). The protein complex shelterin, consisting of TRF1, TRF2, TIN2, POT1, TPP1 and RAP1, binds to telomeric repeats and mediates the formation of a telomeric loop (T-loop) in which the single-stranded $3^{\prime}$-overhang is concealed in a D-loop (Griffith et al., 1999; Doksani et al., 2013). This is necessary to prevent DNA damage response (DDR) and repair mechanisms from recognizing the single-stranded DNA (ssDNA) overhang. Due to incomplete replication of chromosome ends, each round of DNA replication progressively shortens linear chromosomes, risking loss of essential genes or important regulatory regions. To prevent this, telomeres act as a buffer region to maintain genome integrity (Harley et al., 1990). Replication of telomeres is initiated by the polymerase alpha-primase (PP) complex, which consists of subunits that have polymerase and primase activity (Pellegrini, 2012). During lagging-strand synthesis the ultimate RNA primer is removed, but cannot be replaced with DNA, resulting in an overhang. Additionally, leading-strand synthesis creates a transient blunt end that is processed by nucleases to generate a short $3^{\prime}$-overhang. Therefore, incomplete replication of the lagging strand and resection of the leading strand result in $3^{\prime}$-overhang generation, which contributes to telomere shortening and is known as the "end-replication problem" (Chow et al., 2012; Chen and Lingner, 2013; Martinez and Blasco, 2015). Besides the end-replication problem, replication at telomeres is extra challenging because of topological barriers, such as the T-loop and the presence of G-quadruplexes. Proper telomere replication requires G-quadruplex resolution and 
suppression of G-quadruplex formation by the helicases BLM, DNA2, WRN and RTEL1, and also T-loop disassembly by WRN and RTEL1 (Uringa et al., 2012; Vannier et al., 2012, 2013; Crabbe et al., 2004; Edwards et al., 2014; Martinez and Blasco, 2015).

In many stem cells and in the majority of cancer cells telomere shortening is, respectively, partially or completely, compensated by telomerase. Telomerase consists of a telomerase reverse transcriptase (TERT) catalytic subunit and an RNA template (TERC) that add de novo TTAGGG repeats to chromosome ends. Telomerase is recruited to telomeres via TIN2-TPP1, whereby TPP1 promotes telomerase activity and telomere extension. First, the $3^{\prime}$-strand is extended by TERT using TERC as the complementary template to synthesize telomeric repeats. Subsequently, in humans, the CST complex binds to this newly generated $3^{\prime}$-strand and recruits the PP-complex to sequentially fill-in the $5^{\prime}$-strand (Greider and Blackburn, 1985; Reveal et al., 1997; Huang et al., 2012; Nandakumar and Cech, 2013). Alternatively, cancer cells that do not express telomerase can counteract telomere shortening by activating the alternative lengthening of telomeres (ALT) pathway. This pathway makes use of homologous recombination (HR)-dependent exchange/synthesis of telomeric DNA. Telomeric DNA can, for example, be copied from a nearby template (the same telomere or the sister telomere), but also from a more distant template such as a telomere from another chromosome (Pickett and Reddel, 2015). In addition, specialized types of promyelocytic leukemia (PML) bodies, socalled ALT-associated PML bodies (APBs), are essential for telomere maintenance in ALT-positive cells (Yeager et al., 1999). Telomeres cluster in APBs, which in addition to telomerebinding factors and telomeric DNA also contain proteins involved in HR to perform ALT (Pickett and Reddel, 2015). HR is a DNA repair pathway that outside of telomeres is used to correctly repair a DNA break by using the sister chromatid as template.

However, when cells proliferate in the absence of telomerase or ALT, telomeres become critically short and shelterin is not able to bind to chromosome ends in sufficient amounts (Nandakumar and Cech, 2013). This leads to initiation of DDR signaling and DNA repair activities that can impair cell proliferation and harm genome stability (d'Adda di Fagagna et al., 2003; Jacobs and de Lange, 2005; Davoli and de Lange, 2011; Jacobs, 2013). Also, when replication at telomeres stalls because of topological barriers that cannot be resolved by helicases, a DDR is activated to restart replication through HR (Badie et al., 2010; Tacconi and Tarsounas, 2015; Zimmer et al., 2016). The DDR and DNA repair mechanisms at dysfunctional telomeres are tightly regulated by post-translational modifications (PTMs). In addition, telomere maintenance and protection, which function to prevent DDR initiation at telomeres, are also affected by PTMs, including ubiquitination and SUMOylation (Peuscher and Jacobs, 2012).

In the process of ubiquitination, the 76 amino acid protein ubiquitin is covalently conjugated via its C-terminus to the $\varepsilon$-amino group of lysine residues or to the $\mathrm{N}$-terminus of a target protein. Ubiquitination is implicated in many cellular pathways in almost all eukaryotic organisms and can target proteins for proteasomal degradation or affect their activity, localization and interaction with other molecules. The attachment of ubiquitin occurs via an enzymatic cascade consisting of E1 ubiquitinactivating, E2 ubiquitin-conjugating and E3 ubiquitin-ligating enzymes (Ciechanover et al., 1982; Hershko et al., 1983; Komander and Rape, 2012). Moreover, ubiquitin itself can also be ubiquitinated at its $\mathrm{N}$-terminal $\mathrm{M} 1$ residue and at one of its seven internal lysine residues K6, K11, K27, K29, K33, K48 and K63. Therefore, ubiquitin-chains with many different linkages can be formed, significantly increasing their signaling potential and specificity. For example, K48-linked chains usually target proteins for proteasomal degradation (Komander and Rape, 2012). Ubiquitination is reversible through the action of deubiquitinating enzymes (DUBs), of which approximately 100 are known in humans. DUBs are able to cleave off an individual ubiquitin or break the bonds within the ubiquitin-chain, allowing for removal and editing at these sites (Komander et al., 2009).

Another PTM that is very similar to ubiquitination is SUMOylation. In this process, a small ubiquitin-related modifier (SUMO) protein is conjugated to target proteins. This also occurs via an enzymatic cascade, mediated by E1, E2 and E3 SUMO enzymes, which conjugate SUMO to the substrate protein in the same manner as ubiquitin (Johnson, 2004). Additionally, deSUMOylating enzymes can reverse this process (Mukhopadhyay and Dasso, 2007). In contrast to the ubiquitin system, for which over 600 E3 ligases are known to exist in humans, only a few SUMO ligases have been identified so far. In addition, multiple SUMO isoforms exist, with SUMO1 (101 amino acids), SUMO2 (95 amino acids) and SUMO3 (103 amino acids) being the ones that have been studied best (CubenasPotts and Matunis, 2013). In contrast to ubiquitin-chains, SUMO-chains do not directly target proteins for proteasomal degradation, but can prime the target for ubiquitin ligasemediated degradation. Moreover, SUMOylation can influence protein activity, localization and interactions between proteins containing SUMO-interacting motifs (SIMs) (Geiss-Friedlander and Melchior, 2007; Kerscher, 2007). In the past years evidence increased for crucial roles of ubiquitination and SUMOylation in the cellular response to telomere dysfunction that potentially leads to genomic instability. Therefore, the aim of this review is to provide an overview of new findings obtained about ubiquitination and SUMOylation involved in telomere maintenance, replication and dysfunction.

\section{TELOMERE MAINTENANCE: SHELTERIN IN CONTROL}

Aberrant telomere function can have severe cellular consequences by leading to genomic instability, cellular senescence and early apoptosis. Therefore, tightly regulated telomere maintenance is required to ensure protection of chromosome ends. The most significant complex involved in telomere maintenance and protection is shelterin (Figure 1). Shelterin governs telomere maintenance and protection in essentially three main ways: (1) by preventing activation of the DDR and DNA repair mechanisms at telomeres, (2) by facilitating telomere replication and (3) by regulating 


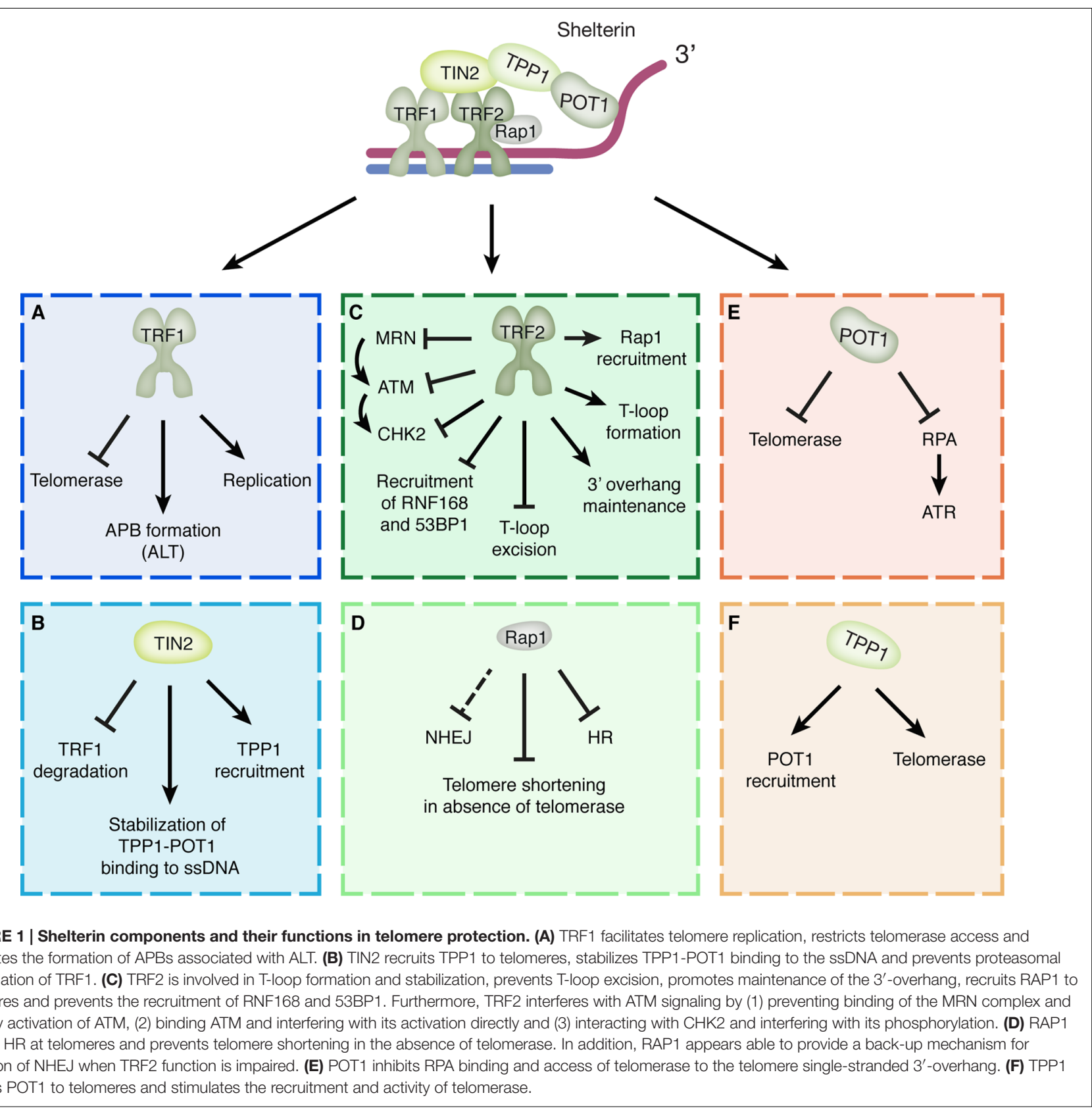

telomerase-mediated telomere elongation. The shelterin components TRF1 and TRF2 directly interact with telomeric DNA and are structurally very similar. Although, both proteins have a TRF homology (TRFH) domain and a SANT/Myb DNAbinding domain, TRF1 and TRF2 do not physically interact and have separate functions (Stewart et al., 2012; Doksani and de Lange, 2014). TRF1 has been shown to be required for proper telomere replication, for example by recruiting the necessary helicases, such as BLM, and for restricting telomerase access to the telomeres (Sfeir et al., 2009). In contrast, TRF2 is involved in T-loop formation and stabilization, prevents T-loop excision and promotes maintenance of the $3^{\prime}$-overhang by recruiting the Apollo nuclease. It is also essential for inhibition of the ATM kinase to repress DNA damage signaling and inhibit classical non-homologous end-joining (c-NHEJ), an error-prone repair pathway that promotes ligation of broken DNA ends (Karlseder et al., 2004; Wang et al., 2004; Denchi and de Lange, 2007; Wu et al., 2010; Doksani et al., 2013; Okamoto et al., 2013). TRF2 interacts with the shelterin component RAP1 and recruits it to the telomeres. Unlike for TRF2, the contribution of RAP1 to protection of mammalian telomeres against NHEJ is less evident and only noticeable in experimental conditions where RAP1 is artificially recruited to TRF2-depleted telomeres or TRF2 function is partially compromised (Sarthy et al., 2009; Kabir et al., 2014; BenarrochPopivker et al., 2016). A more obvious role for RAP1 appears 
to be in protecting telomeres against HR. RAP1 deletion in a $\mathrm{Ku} 70^{-/-}$background resulted in increased telomere-sister chromatid exchanges, indicating that RAP1 represses HR (Sfeir et al., 2010). Furthermore, telomeres devoid of RAP1 and the N-terminal basic domain of TRF2 are rapidly resected by HR factors, resulting in telomere loss and telomere-free fusions (Rai et al., 2016). Finally, mice lacking both RAP1 and telomerase show increased telomere shortening and progressively decreased survival compared to single telomerase knockout mice (Martinez et al., 2016). Thus RAP1 seems to aid both in protecting telomeres from DNA repair activities and in maintaining telomeres in absence of telomerase. Much of the mechanistic basis for these roles of RAP1 still remains to be discovered.

TRF1 and TRF2 are bridged by TIN2, which acts as a linker protein. This ensures the integrity of the whole shelterin complex by connecting not only the double-strand DNA-binding TRF1 and TRF2, but also by linking TRF1 and TRF2 to the ssDNAbinding TPP1-POT1 heterodimer. In addition, TIN2 also recruits TPP1 to the telomere and stabilizes TPP1-POT1 binding to the ssDNA (O'Connor et al., 2006; Palm and de Lange, 2008). POT1 is the only shelterin component that directly interacts with the single-stranded $3^{\prime}$-overhang through its two OB-fold domains (Zhong et al., 2012). It is recruited to the telomeres by interacting with TPP1 (Loayza and De Lange, 2003; Liu et al., 2004). Binding of the POT1-TPP1 complex to telomeres contributes to telomere maintenance by preventing other factors, such as replication protein A (RPA), from binding to the $3^{\prime}$-overhang and promoting DNA damage signaling. In addition, both POT1 and TPP1 are involved in the regulation of telomerase activity, showing opposing effects on telomerase (Stewart et al., 2012). Whereas POT1 negatively regulates telomerase binding by making the 3 '-overhang inaccessible, TPP1 has been observed to promote recruitment of telomerase and stimulate its activity (Stewart et al., 2012). Although it is not yet exactly known how these opposing functions of POT1 and TPP1 are coordinated, recent work suggests that POT1 inhibits telomerase recruitment by suppressing phosphorylation of TPP1 at Ser255 by the M-phase kinase NEK6 (Hirai et al., 2016). According to the proposed model, POT1 might dissociate from the telomeres during replication, after the T-loop is dismantled, thereby relieving the inhibitory effect of POT1 on TPP1. This could then allow for phosphorylation of TPP1 and thereby promote recruitment of telomerase to telomeres to maintain the telomeric sequence (Hirai et al., 2016).

\section{Regulation of TRF1 by Ubiquitin}

In recent years, shelterin components have been shown to be regulated by PTMs. TRF1 levels are regulated by ubiquitinmediated degradation that is facilitated by three E3 ligases: RLIM (RING H2 zinc finger or RNF12) and the F-box proteins FBX4 and $\beta$-TRCP1 (Lee et al., 2006; Her and Chung, 2009; Wang C. et al., 2013) (Figure 2). RLIM binds to a region between the dimerization and Myb domain of TRF1 and targets TRF1 for proteasomal degradation (Her and Chung, 2009). Similarly, FBX4 binds to the N-terminal region of the TRFH dimerization domain of free TRF1 (unbound to telomeric DNA) and also targets TRF1 for proteasomal degradation (Lee et al., 2006). When either RLIM or FBX4 is depleted TRF1 levels are stabilized, resulting in impaired cell growth and a decrease in telomere length, as TRF1 binding to telomeres is inhibitory toward telomerase. Accordingly, upon RLIM or FBX4 overexpression, levels of TRF1 decline, indicating a negative regulatory role for RLIM and FBX4 on TRF1 (Her and Chung, 2009). Moreover, a recent study has identified a novel TRF1-interacting protein that prevents FBX4 binding to TRF1. The splicing factor U2AF65 acts as a positive regulator of TRF1 by preventing FBX4-mediated ubiquitination and subsequent degradation of TRF1 (Kim and Chung, 2014). It has been proposed that U2AF65 only interacts with telomere unbound TRF1, as U2AF65 interacts with the Myb domain of TRF1 that is used by TRF1 to bind telomeric DNA. The Myb domain of TRF1 would therefore be inaccessible to U2AF65 when TRF1 is bound to DNA. Although U2AF65 interacts with a different domain of TRF1 than FBX4, which interacts with the TRFH domain of TRF1, TRF1 cannot interact with both proteins simultaneously (Kim and Chung, 2014). In addition, the shelterin component TIN2 also interferes with FBX4-mediated TRF1 turnover. TIN2 interacts with the TRFH dimerization domain of TRF1 (Ye and de Lange, 2004), preventing FBX4 association and thereby TRF1 ubiquitination and subsequent degradation (Zeng et al., 2010). TIN2 itself is also affected by ubiquitination. Its turnover is regulated by the E3 ligase SIAH2, which interacts with TIN2 to facilitate its proteasomal degradation (Bhanot and Smith, 2012). Finally, the F-box protein $\beta$-TRCP1 has also been shown to interact with TRF1 and promote its degradation. Similar to RLIM and FBX4, $\beta$-TRCP1 overexpression results in a reduced half-life of TRF1, while $\beta$-TRCP1 depletion leads to stabilization of TRF1 (Wang C. et al., 2013). Interestingly, $\beta$-TRCP1 overexpression also resulted in an increase in the percentage of APBs, which is surprising as TRF1 is known to be required for APB formation. Although an explanation could be that perhaps $\beta$-TRCP1 degrades not all but only a specific pool of TRF1, further studies are necessary to determine how TRF1 degradation by $\beta$-TRCP1 can be correlated with a function for $\beta$-TRCP1 in APB formation.

In contrast to the E3 ligases RLIM, FBX4 and $\beta$-TRCP1 promoting TRF1 turnover, the GCN5 and USP22 components of the chromatin modifying complex SAGA have been shown to oppose TRF1 ubiquitination (Atanassov et al., 2009). Depletion of either the histone acetyltransferase GCN5 or the DUB USP22 results in a decrease in TRF1 levels, which can be prevented by inhibition of the proteasome. GCN5 was found to be required for USP22 to properly associate with the SAGA complex and to be able to deubiquitinate TRF1 and prevent its turnover (Atanassov et al., 2009). In conclusion, the above-discussed studies indicate that TRF1 levels in cells are tightly regulated by numerous different proteins and on multiple levels.

\section{Ubiquitination- and SUMOylation-Mediated Regulation of TPP1, TRF2 and RAP1}

Another shelterin subunit that is subjected to ubiquitin-mediated proteolysis is TPP1, which is evidenced by stabilization of TPP1 
A

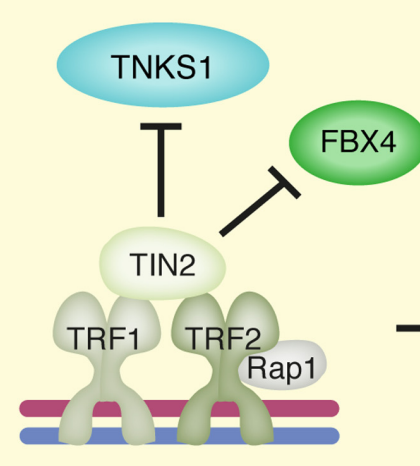

C



D

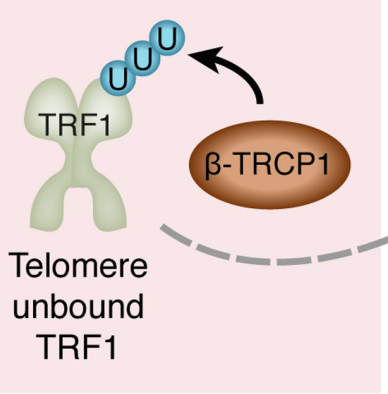

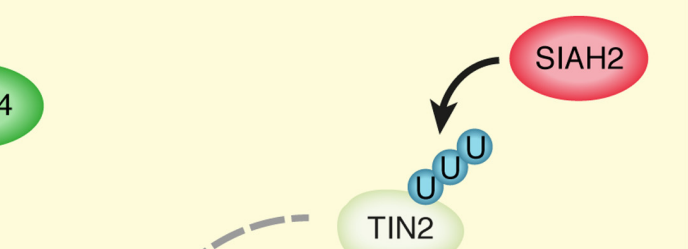

B

r
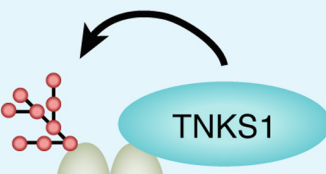

TRF1

$-=$
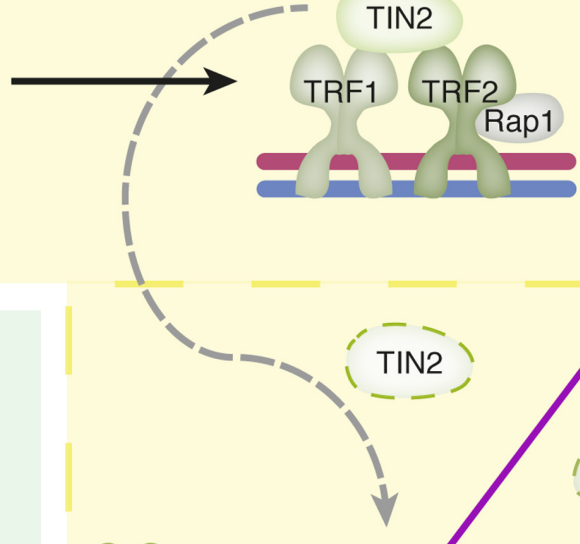

(2)



Proteasomal
degradation
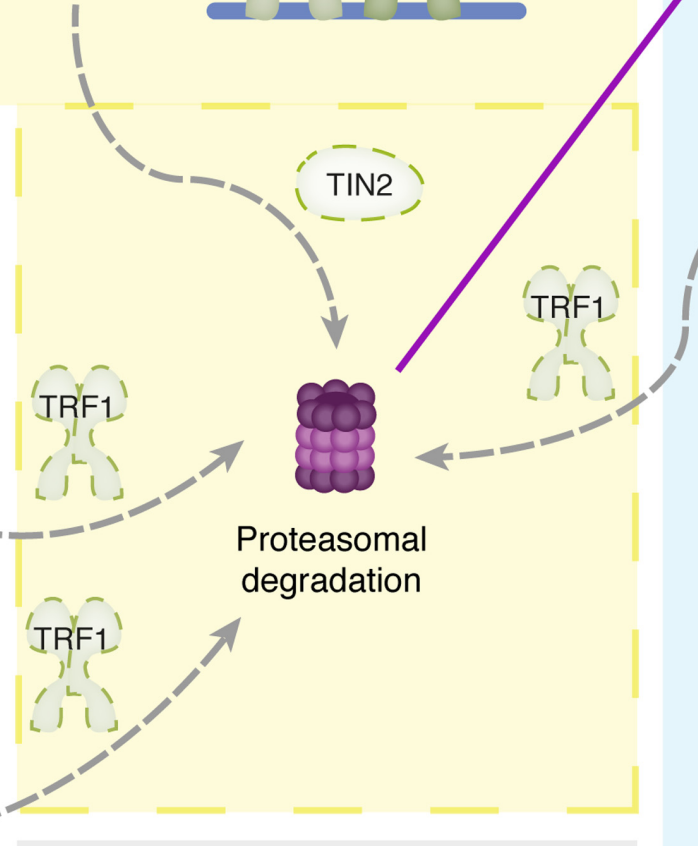

1



Telomere unbound TRF1

Q PARylation

(U) Ubiquitination

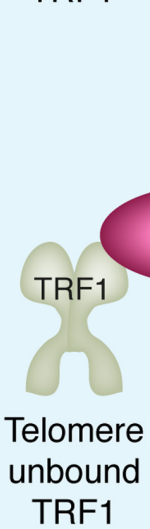

FIGURE 2 | The multilevel regulation of TRF1 degradation. (A) TIN2 protects TRF1 from proteasomal degradation by preventing binding of Tankyrase 1 (TNKS1) and the E3 ligase FBX4 to TRF1. However, TIN2 itself is also targeted for proteasomal degradation by ubiquitination through the E3 ligase SIAH2, which releases the inhibition on TNKS1 and FBX4. (B) Subsequently, TNKS1 can PARylate TRF1 resulting in its dissociation from the telomeric DNA. This allows for FBX4-mediated TRF1 ubiquitination and proteasomal degradation. However, U2AF65 also binds telomere-unbound TRF1, which inhibits FBX4 binding and subsequent TRF1 degradation. (C,D) The E3 ligases RLIM (C) and $\beta$-TRCP1 (D) also ubiquitinate telomere-unbound TRF1 and promote its proteolysis.

protein levels upon inhibition of the proteasome. Although the E3 ubiquitin ligases targeting TPP1 are still unknown, the DUB USP7 has been shown to interact with human TPP1 and to remove ubiquitin chains from its surface. While USP7 depletion did not affect proteasome-regulated TPP1 levels, USP7 might interact in a redundant manner with other DUBs to stabilize TPP1 (Zemp and Lingner, 2014). In mice, TPP1 ubiquitination by the E3 ligase RNF8 is also required for its stabilization at telomeres (Rai et al., 2011). However, in humans such a regulatory role of ubiquitination on TPP1, beyond regulation of its turnover, has not been observed. Changes in ubiquitination of TPP1 in humans have not resulted in aberrant TPP1 function nor have shown effects on TPP1 interaction with other proteins, such as TIN2, POT1 and telomerase (Zemp and Lingner, 2014). While this could be related to the use of overexpressed tagged TPP1 in human cells, it might also potentially indicate species differences in the extent of regulatory roles of ubiquitination on TPP1. Nevertheless, additional roles of human TPP1 ubiquitination may still await discovery.

Furthermore, the shelterin subunit TRF2 has also been shown to be ubiquitinated. TRF2 turnover is regulated by the E3 ligase SIAH1 as part of a positive feedback loop involving TRF2, ATM and p53 (Fujita et al., 2010). When telomere shortening causes loss of TRF2-mediated telomere protection, the ATM kinase is 
activated, which induces p53 activity and results in replicative senescence. Subsequently, p53 induces transcription of SIAH1, which targets TRF2 for proteasomal degradation. This results in increased p53 activation, further decreasing TRF2 levels through SIAH1-mediated ubiquitination of TRF2 (Fujita et al., 2010). In addition, a crosstalk between ubiquitination and SUMOylation has recently been observed to contribute to regulation of TRF2 (Her et al., 2015). The E3 SUMO ligase PIAS1 was identified as a novel TRF2-interacting protein and shown to SUMOylate TRF2. SUMOylated TRF2 is subsequently recognized by the SUMOtargeted ubiquitin ligase (STUbL) RNF4 through its SIM. This results in ubiquitination of TRF2 and subsequent proteasomal degradation (Her et al., 2015). This probably affects only a fraction of the total pool of TRF 2 in the cell, as TRF2 is essential for chromosome end protection and extensive turnover of TRF2 would result in telomere uncapping.

Finally, in Saccharomyces cerevisiae (budding yeast) Rap1 has been shown to be SUMOylated and subsequently targeted for proteasomal degradation by the STUbL Uls1 (Lescasse et al., 2013). Loss of Uls1 was shown to result in accumulation of poly-SUMOylated Rap1 and telomere fusions. These fusions could be prevented by introduction of rap1 alleles lacking SUMOylation sites. This indicates that accumulation of polySUMOylated Rap1 promotes telomere fusion and suggests that poly-SUMOylated Rap1 is non-functional in telomere protection. The proposed model suggests that Uls1 promotes ubiquitination and subsequent degradation of poly-SUMOylated Rap1, thereby allowing for recruitment of non-SUMOylated Rap1 that is able to protect chromosome ends from fusing through NHEJ (Lescasse et al., 2013). To what extent these results can be translated to mammalian systems remains unclear, as in budding yeast Rap1 interacts directly with telomeres and protects against NHEJ, while in mammals no direct interaction between RAP1 and telomeres is detectable but RAP1 is recruited by TRF2 and seems to mainly protect against $\mathrm{HR}$.

Altogether, it has become clear that telomere maintenance does not only depend on the binding-capability of shelterin itself to the telomeric DNA, but also on its regulation by PTMs. Both features are important in facilitating protection of genome stability by telomeres. Although ubiquitination has been shown to contribute to telomere maintenance in multiple ways, emerging data show that SUMOylation also plays an important role in this process. Further studies are likely to provide additional insight in how these modifications affect and regulate telomere function. Additionally, it would be beneficial to verify the extent to which findings from yeast studies are conserved in mammalian systems.

\section{TELOMERE ELONGATION}

The majority of cancer cells $( \pm 90 \%)$, as well as many stem cells, express telomerase to elongate telomeres (Lazzerini-Denchi and Sfeir, 2016). Recently, it has been shown that in human embryonic stem cells (hESCs) telomere length is stabilized by a tight balance between telomere elongation through telomerase and telomere trimming by XRCC3 and NBS1 (Rivera et al.,
2017). In tumors, telomere length is less stable, as telomere length between cancer cells is variable and telomerase extends most of the telomeres during every replication cycle (Martinez and Blasco, 2015). Although the majority of cancer cells maintains telomere length by activation of telomerase, a smaller number uses the ALT mechanism. During ALT, a HR-dependent mechanism copies telomeric DNA from a nearby template, resulting in telomere lengthening but also telomeres loss, which account for the heterogeneous telomere length typically observed in ALT cells (Pickett and Reddel, 2015).

Telomerase and ALT have been shown to be regulated by various PTMs, including ubiquitination and SUMOylation. Below we will discuss the roles of these two PTMs in regulation of telomerase activity and stability, and in ALT.

\section{Telomerase in the Spotlight}

Especially the TERT subunit of telomerase has been shown to be modified by multiple ubiquitin E3 ligases, most of them regulating its proteasomal degradation (Figure 3 ). The first E3 ubiquitin ligase that was identified to interact with and ubiquitinate human (h) TERT is MKRN1 (Makorin-1 or RNF61) (Kim et al., 2005). Overexpression of this E3 ligase was shown to decrease telomerase activity and telomere length through ubiquitination and subsequent degradation of hTERT. In addition, MKRN1 has also been specifically implicated in modulation of telomerase activity during cell differentiation (Salvatico et al., 2010). The cancer cell line HL60 normally expresses the MKRN1 gene at very low levels and MKRN1 protein levels cannot be detected. However, upon retinoic acid induced differentiation of HL-60 cells MKRN1 expression significantly increased, coinciding with a strong down regulation of telomerase activity. As hTERT has a long half-life, MKRN1-mediated degradation of hTERT could provide efficient degradation of hTERT when telomerase activity is no longer needed (Salvatico et al., 2010).

A second E3 ubiquitin ligase that directly acts on hTERT is MDM2 (also known as HDM2). MDM2 and hTERT can physically interact through multiple domains on both proteins, whereby hTERT is polyubiquitinated and degraded by the proteasome. In line with this, depletion of MDM2 resulted in increased hTERT protein levels and increased telomerase activity (Oh et al., 2010). In addition, the E2 ubiquitinconjugating enzyme UBE2D3 (or UBCH5C) has also been shown to regulate hTERT ubiquitination. Similar to MDM2 depletion, UBE2D3 depletion results in hTERT accumulation and increased telomerase activity (Wang W. et al., 2013; Gao et al., 2016; Yang et al., 2016). As it is known that UBE2D3 and MDM2 function together in the ubiquitination of p53 (Saville et al., 2004) and both were shown to regulate hTERT ubiquitination, it is tempting to speculate that this E2-E3 couple might also act together in the ubiquitination of hTERT.

A third E3 ligase that interacts with hTERT is the co-chaperone protein CHIP (C-terminus of Hsc70-interacting protein). In contrast to the ligases mentioned above, CHIP binds to the premature form of hTERT in the cytoplasm to inhibit its transport into the nucleus and subsequent integration into the telomerase complex. This inhibitory function of CHIP on the 


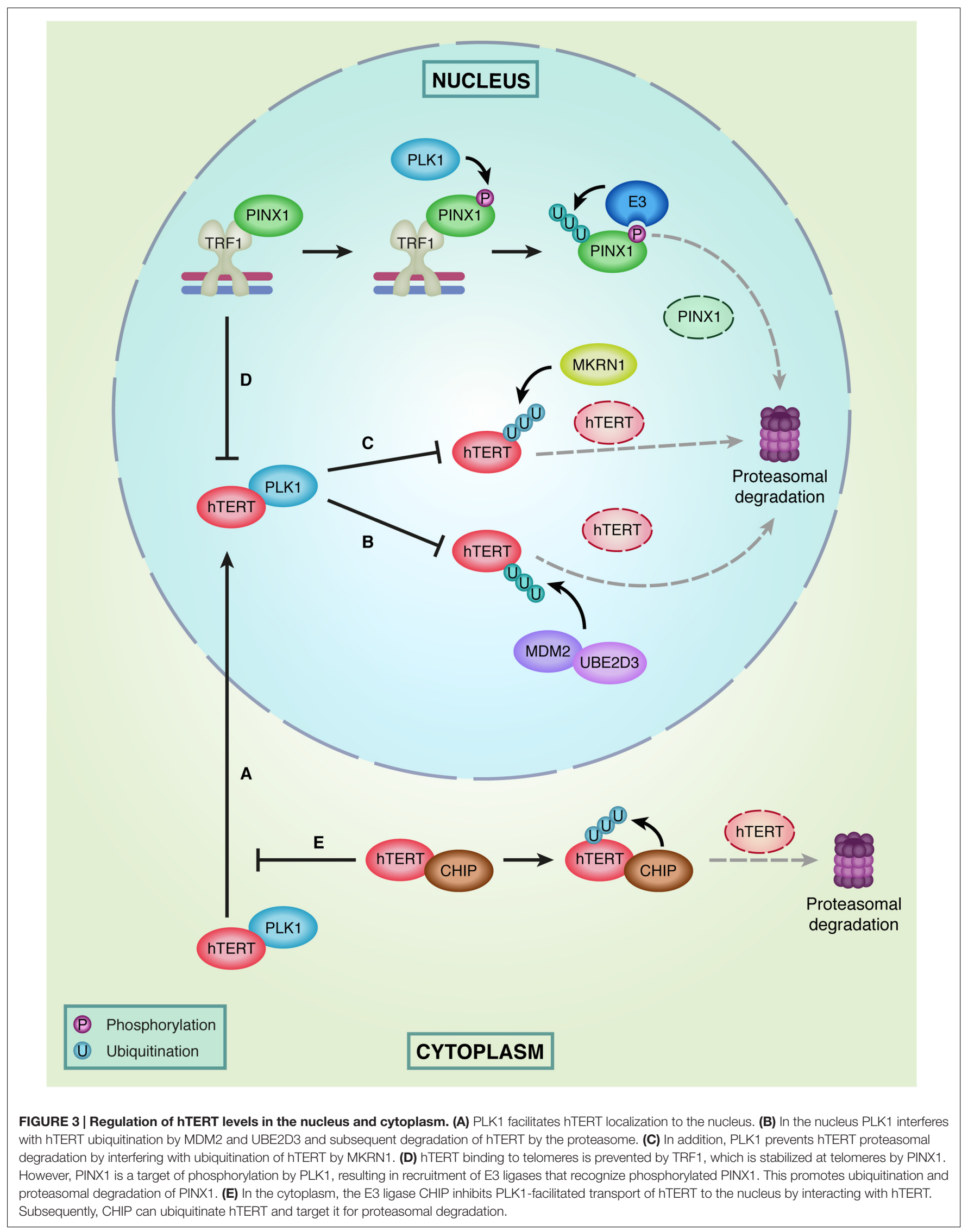


nuclear import of hTERT has been shown to occur independently from the ubiquitin ligase activity of CHIP, which requires its U-box domain. However, for ubiquitination and subsequent degradation of hTERT, the U-box and E3 ligase activity of CHIP are necessary (Lee et al., 2010). Furthermore, the interaction between CHIP and hTERT was found to peak in G2/M phase and decrease during S-phase, suggesting that degradation of hTERT by CHIP is cell cycle regulated and exerted when telomerase does not act on telomeres.

In addition to MKRN1, MDM2 and CHIP, Polo-like kinase 1 (PLK1) was recently identified to directly interact with hTERT, but with positive effects on hTERT levels (Huang et al., 2015). PLK1 overexpression was shown to prevent ubiquitination and proteasomal degradation of hTERT and to result in increased total protein levels of hTERT, including increased levels of nuclear hTERT and chromatin-bound hTERT. Therefore, it was suggested that PLK1 facilitates localization of hTERT to the nucleus and might interfere with the function or binding of E3 ligases, such as MKRN1 and MDM2, to hTERT. This then prevents hTERT's proteasomal degradation and stabilizes telomerase activity (Huang et al., 2015). Interestingly, elevated PLK1 expression has been observed in several tumors. This might contribute to increased hTERT levels and excessive telomerase activity in cancer cells, thereby increasing the proliferative capacity of these cells (Holtrich et al., 1994).

Additionally, PLK1 has also been implicated in mediating the turnover of PINX1 (Wang et al., 2010). PINX1 is known as an hTERT inhibitor that directly interacts with TRF1 (Zhou and Lu, 2001). PINX1 binding to TRF1 has been shown to promote TRF1 association with telomeric DNA, thereby contributing to telomerase inhibition by decreasing accessibility of telomeric DNA to telomerase (Yoo et al., 2009). PLK1 can phosphorylate PINX1 and induce its ubiquitinmediated proteasomal degradation. It has been proposed that phosphorylation of PINX1 by PLK1 stimulates the activity of E3 ubiquitin ligases, which subsequently ubiquitinate PINX1 and target it for degradation (Wang et al., 2010). When PINX1 is degraded, other factors can gain access to TRF1 and might affect the binding of TRF1 to telomeres, thereby increasing telomerase recruitment. One of these factors is the poly (ADPribose) polymerase Tankyrase 1 (TNKS1). TNKS1 has been shown to interact with TRF1 and to PARylate it, resulting in TRF1 dissociation from telomeres and subsequent degradation of TRF1 by the E3 ligase FBX4 (Smith et al., 1998; Ye and de Lange, 2004; Lee et al., 2006) (Figure 2). Consequently, telomerase gains access to telomeres, leading to telomere elongation. Interestingly, PLK1 can also phosphorylate TNKS1 directly, which stabilizes TNKS1 protein levels and thereby increases TRF1 degradation and telomerase activity ( $\mathrm{Ha}$ et al., 2012). The shelterin protein TIN2 adds a final layer of complexity to this, as TIN2 can interact with TNKS1 and TRF1, thereby preventing TRF1 inactivation by TNKS1, resulting in TRF1 accumulation at telomeres and inhibition of telomerase loading (Ye and de Lange, 2004).

While ubiquitination evidently plays an important role in the regulation of telomerase activity in mammals, the contribution of SUMOylation to regulation of mammalian telomerase is not yet evident, despite the discovery of
SUMOylation-dependent mechanisms of telomerase control in yeast. In Schizosaccharomyces pombe (fission yeast) SUMOylation of Tpz1, the homolog of TPP1, has been connected to negative regulation of telomere elongation (Hang et al., 2011; Garg et al., 2014; Miyagawa et al., 2014). SUMOylation of Tpz1 decreases telomerase binding to telomeres. This occurs through recruitment of the Stn1-Ten1 subunits of the CST complex, known to be involved in telomere length regulation and chromosome end capping by preventing telomerase accumulation at telomeres (Price et al., 2010). In contrast, Tpz1 has also been shown to interact with Ccq1-Est1 and thereby promote the recruitment and activity of telomerase, indicating a double role for Tpz1 in maintaining telomere length homeostasis (Miyoshi et al., 2008; Miyagawa et al., 2014). In budding yeast, the Stn1 protein has also been observed as a negative regulator of telomerase activity and telomere elongation. Stn 1 interacts with the POT1 homolog Cdc13 and this interaction is increased when Cdc13 is SUMOylated, strengthening the inhibitory effect of Stn 1 on telomerase. This increased interaction between Stn1 and $\mathrm{Cdc13}$ has been associated with reduced telomerase levels, supporting a negative regulatory effect of SUMOylation on telomerase activity (Hang et al., 2011).

In conclusion, multiple different proteins and posttranslational modifications, including ubiquitination, phosphorylation and PARylation, directly or indirectly affect the activity of telomerase. Whether there is also a role for SUMOylation in the control of telomerase regulation in mammalian cells still needs to be uncovered.

\section{Alternative Lengthening of Telomeres: Surviving without Telomerase}

Ubiquitination and SUMOylation are also important for promoting telomere elongation by ALT in cells without telomerase activity. The shelterin protein TRF1 has been shown to be an important factor for ALT by promoting APB formation and localization of telomeres to PML bodies. To be able to facilitate this, TRF1 needs to be SUMOylated by MMS21, the E3 SUMO ligase component of the SMC5/6 complex (Potts and Yu, 2007). If MMS21-mediated SUMOylation is prevented, TRF1 recruitment to PML bodies is inhibited and APB formation is impaired. In addition to TRF1, also TRF2, TIN2 and RAP1 are SUMOylated by MMS21. When MMS21 is depleted, SUMOylation of all these shelterin subunits is inhibited (Potts and Yu, 2007). SUMOylation of multiple components of a complex could affect its stability; therefore, MMS21-mediated SUMOylation of shelterin components was suggested to induce disassembly of the shelterin complex within APBs. This could result in telomere deprotection, thereby potentially facilitating telomere recombination in ALT cells. As mentioned before, TRF1 can also be ubiquitinated by the E3 ligase $\beta$-TRCP1, promoting degradation of TRF1. However, $\beta$-TRCP1 has also been found to be essential for APB formation, as inhibition of $\beta$-TRCP1 decreased the percentage of APBs (Wang C. et al., 2013). This seems contradictory for a negative regulator of TRF1, but it has been suggested that $\beta$-TRCP1 only degrades telomere-unbound TRF1. If SUMOylation of the shelterin subunits indeed results in 
disassembly of the shelterin complex, $\beta$-TRCP1 might assist in the degradation of telomere-unbound TRF1.

Another factor involved in ALT is the scaffold protein SLX4, which recognizes DNA lesions and facilitates DNA repair by interacting with multiple endonucleases. Its structure includes both ubiquitin-binding zinc fingers (UBZs), involved in DNA interstrand crosslink repair, as well as SIMs that are important for localization of SLX4 to ALT telomeres (Ouyang et al., 2015). In addition, SLX4 contributes to telomere maintenance and protection by directly interacting with TRF2 via a motif resembling the TRF2-binding motif (TBM) present in other proteins known to interact with TRF2 (Wilson et al., 2013). The SUMO binding capacity of SLX4 was shown to enhance its interaction with several DNA damage sensors and telomerebinding proteins, including RPA, the MRE11-RAD50-NBS1 (MRN) complex and TRF2 (Ouyang et al., 2015). Therefore, increased SUMOylation of proteins at ALT telomeres may assist in the recruitment of SLX4 and other factors involved in ALT.

In budding yeast, telomere-bound proteins become increasingly SUMOylated when cells without telomerase activity obtain critically short telomeres that induce crisis. These SUMOylated proteins are recognized by the STUbL Slx5-Slx8, which mediates the relocalization of critically short telomeres to nuclear pore complexes (NPCs), where recombination events similar to the mammalian ALT pathway occur (Churikov et al., 2016). As Slx5-Slx8 can interact with SUMOylated telomere-bound proteins, as well as with the Nup84 complex of NPCs, it is believed to tether telomeres to the NPCs, thereby enabling recombination (Nagai et al., 2008; Churikov et al., 2016). Interestingly RNF4, the human homolog of Slx5-Slx8, has been shown to localize to PML bodies in human cells (Lallemand-Breitenbach et al., 2008; Tatham et al., 2008; Weisshaar et al., 2008). Although it is unknown whether RNF4 activity is necessary for ALT, it would be interesting to investigate if RNF4 has a similar function in humans as Slx5-Slx8 has in budding yeast. As discussed above, the MMS21 component of the SMC5/6 complex is necessary for SUMOylation at telomeres, which is needed for the recruitment of telomeres to PML bodies. RNF4 could potentially be involved in this by recognizing the MMS21-SUMOylated TRF1 at telomeres and promoting telomere recruitment to APBs.

Altogether, the above-discussed data show that ubiquitination and SUMOylation are crucial in the regulation of telomere elongation by telomerase and ALT. Multiple E3 ligases were shown to control the ubiquitination of hTERT to tightly regulate its levels and activity. In addition, various proteins and PTMs regulate the inhibitory function of TRF1 on telomerase. The complexity of telomerase regulation at telomeres suggests that this regulation is strictly controlled to prevent unscheduled access of telomerase to telomeres. However, further studies are necessary to understand how these mechanisms are coordinated and whether they are interrelated. In addition, ubiquitination and SUMOylation of shelterin components also seems to be crucial in promoting ALT, indicating that posttranslational modification of shelterin components contributes to multiple processes involved in telomere maintenance and elongation.

\section{TELOMERE DEPROTECTION}

Successive rounds of cell division in the absence of telomerase or ALT ultimately lead to critically short telomeres and deprotected chromosome ends. These deprotected ends are recognized as damaged DNA by the DDR machinery. This results in activation of the p53 and Rb pathways and entry into senescence to limit telomere fusions and prevent subsequent genomic instability. However, when these pathways are impaired, senescence is bypassed and cells continue to divide and further lose telomere repeats until they reach crisis, a state of massive genome instability and cell death (d'Adda di Fagagna et al., 2003; Jacobs and de Lange, 2005; Palm and de Lange, 2008). At this stage, DNA repair pathways are activated and telomeres fuse in G1-phase either through Artemis and DNA ligase IV mediated c-NHEJ, or through alternative NHEJ (alt-NHEJ), a pathway mediated by PARP1 and DNA Ligase III. During subsequent cell divisions breakage-fusion-bridge cycles occur, inducing genomic instability and cell death. Nevertheless, a small portion of cells might escape crisis by reactivating telomerase or inducing ALT to maintain their telomeres, which results in expansion of cells with aberrant genomes, thereby promoting tumorigenesis (Arnoult and Karlseder, 2015; Lazzerini-Denchi and Sfeir, 2016).

\section{Signaling through the RNF8-RNF168 Pathway at Uncapped Telomeres}

The DDR activated by deprotected telomeres in many ways resembles the DDR at general DNA double-strand breaks (DSBs). The DDR at telomeres starts with the recognition of uncapped telomeres by the MRN complex and activation of the ATM kinase, resulting in phosphorylation of histone $\mathrm{H} 2 \mathrm{AX}$ at serine 139, generating $\gamma \mathrm{H} 2 \mathrm{AX}$. This serves as a binding platform for MDC1, which initiates a positive feedback loop by promoting further accumulation of MRN and ATM (Peuscher and Jacobs, 2012). This results in spreading of $\gamma \mathrm{H} 2 \mathrm{AX}$ along the chromatin and amplification of DDR signaling and repair factor recruitment (Cimprich and Cortez, 2008; Brown and Jackson, 2015). At DSBs MDC1 is known to recruit RNF8, which interacts with phosphorylated MDC1 via its FHA domain (Kolas et al., 2007; Mailand et al., 2007). Depletion of either MDC1 or RNF8 causes a similar defect in the accumulation of 53BP1 at dysfunctional telomeres and reduces telomere fusions upon TRF2 inhibition (Dimitrova and de Lange, 2006; Peuscher and Jacobs, 2011). Furthermore, RNF8 requires its FHA domain to accumulate and promote NHEJ at uncapped telomeres, suggesting that RNF8 also recognizes phosphorylated MDC1 in this setting. Together this indicates that MDC1 and RNF8 function in the same pathway at telomeres and in a way that is identical to the DDR at genome-wide DSBs (Peuscher and Jacobs, 2011).

Upon RNF8 recruitment to uncapped telomeres, the RNF8/RNF168 signaling cascade is activated, promoting ubiquitination of histone $\mathrm{H} 2 \mathrm{~A}$ and subsequent recruitment of $53 \mathrm{BP} 1$. In addition, the recruitment of $53 \mathrm{BP} 1$ is dependent on the recognition of $\mathrm{H} 4 \mathrm{~K} 20 \mathrm{me} 2$ by the Tudor domains of $53 \mathrm{BP} 1$ 
(Dimitrova et al., 2008). 53BP1 recruitment to telomeres results in accrual of RIF1 and MAD2L2 to promote NHEJ. This has been shown to block $5^{\prime}$ end-resection and HR through inhibition of BRCA1 recruitment to uncapped telomeres (Dimitrova et al., 2008; Chapman et al., 2013; Zimmermann et al., 2013; Boersma et al., 2015). The same mechanism has also been described at DNA DSBs and in immunoglobulin class-switch recombination (CSR) (Manis et al., 2004; Bothmer et al., 2010; Di Virgilio et al., 2013; Escribano-Diaz et al., 2013; Feng et al., 2013; Boersma et al., 2015; Xu et al., 2015). In addition, 53BP1 also recruits PTIP, which was also reported to contribute to fusion of dysfunctional telomeres. Furthermore, in BRCA1 deficient cells PTIP was shown to inhibit DSB resection and thereby promote genomic instability (Callen et al., 2013).

Depletion of RNF8 in cells with inactivated TRF2 leads to decreased $\mathrm{H} 2 \mathrm{~A}$ ubiquitination and 53BP1 recruitment at telomeres and a reduction in telomere fusions (Peuscher and Jacobs, 2011). In addition, inhibition of RNF168 recruitment to telomeres by the iDDR domain of TRF2 (part of the hinge domain) also results in a decrease in 53BP1 accumulation at dysfunctional telomeres (Okamoto et al., 2013). It has been shown that the iDDR domain of TRF2 prevents RNF168 recruitment to telomeres by accrual of the E3 ubiquitin ligase UBR5 and the MRN complex, resulting in inhibition of the signaling cascade downstream of ATM and protecting against telomere fusions through inhibition of NHEJ. UBR5 has been shown to function together with the E3 ligase TRIP12 at DSBs to control the levels and recruitment of RNF168 by targeting it for proteasomal degradation (Gudjonsson et al., 2012). The interaction of TRF2 with UBR5 could therefore inhibit RNF168 recruitment to telomeres. Interestingly, the MRN complex was shown to recruit the DUB BRCC3, which is part of the BRCA1-A complex and has been suggested to counteract the action of RNF8-UBC13 at DSBs (Shao et al., 2009). In this way, BRCC3 could inhibit recruitment of RNF168 to telomeres. Although BRCA1 is usually implicated in facilitating end-resection and HR, the BRCA1-A complex has also been suggested to restrict end-resection at DNA breaks (Coleman and Greenberg, 2011). In addition, BRCA1 has been shown to contribute to chromosome end protection (Al-Wahiby and Slijepcevic, 2005). Altogether, these studies indicate that also at dysfunctional telomeres the RNF8/RNF168 pathway promotes $\mathrm{H} 2 \mathrm{~A}$ ubiquitination and recruitment of $53 \mathrm{BP} 1$ to activate NHEJ.

Recently, RNF8 has been shown to ubiquitinate histone $\mathrm{H} 1$ at DSBs, together with the E2 ubiquitin-conjugating enzyme UBC13 (Thorslund et al., 2015). Ubiquitinated histone $\mathrm{H} 1$ serves as a binding platform for RNF168, which subsequently ubiquitinates histone $\mathrm{H} 2 \mathrm{~A}$ on $\mathrm{K} 13 / \mathrm{K} 15$ and allows for recruitment of several repair factors, such as 53BP1 and BRCA1 (Mattiroli et al., 2012; Fradet-Turcotte et al., 2013; Thorslund et al., 2015). Furthermore, the DUB USP51 was shown to reverse the ubiquitination of histone $\mathrm{H} 2 \mathrm{~A}$ at $\mathrm{K} 13 / \mathrm{K} 15$. Depletion of USP51 induces an increase in H2A K13/K15 ubiquitination and a delay in DDR foci resolution (Wang et al., 2016). However, many other DUBs have also been shown to be able to deubiquitinate histone $\mathrm{H} 2 \mathrm{~A}$, including USP3,
USP16, USP26 and USP44 (Citterio, 2015). Whether RNF8 ubiquitinates histone $\mathrm{H} 1$ at telomeres and whether RNF168 modifies histone $\mathrm{H} 2 \mathrm{~A}$ at $\mathrm{K} 13 / \mathrm{K} 15$ at telomeres remains to be elucidated.

Finally, the STUbL RNF4 has also been shown to promote NHEJ at uncapped telomeres (Groocock et al., 2014). It usually recognizes SUMO-modified targets and is only activated upon dimerization in the presence of SUMO-chains (RojasFernandez et al., 2014). RNF4 was suggested to promote 53BP1 recruitment to uncapped telomeres and telomere fusions, depending on a nucleosome-targeting motif in its RING domain and its SIMs (Groocock et al., 2014). This suggests that RNF4 recognizes chromatin-bound SUMO conjugates via its SIM domains (interaction with SUMO proteins) and its RING domain (binding to chromatin) and can subsequently ubiquitinate nearby chromatin or target proteins.

\section{Repression of the DDR and DNA Repair at Telomeres}

In contrast to repair at genome-wide DSBs, which contributes to genome stability by fixing the break, repair at uncapped telomeres can be deleterious. When DNA repair factors gain access to chromosome ends and create end-to-end fusions in an attempt to 'heal the break', this can result in genomic instability. Therefore, telomeres need to be protected from unwanted actions of DNA repair factors. This protection is mainly achieved by the TRF2 and POT1 subunits of the shelterin complex (Doksani and de Lange, 2014). TRF2 has been shown to protect telomeres in at least five ways. First, TRF2 binding to telomeric DNA stimulates strand invasion and thereby T-loop formation, which hides telomere ends and prevents binding of the MRN complex and subsequent activation of ATM (Griffith et al., 1999; Stansel et al., 2001; Doksani et al., 2013). Secondly, TRF2 interferes directly with activation of the ATM kinase. TRF2 was found to be able to bind to ATM and prevent its phosphorylation at S1981, resulting in inhibition of the signaling cascade downstream of ATM (Karlseder et al., 2004). Thirdly, TRF2 has been shown to interact with the ATM target CHK2 at a position close to its Thr68 phosphorylation site, preventing activation of CHK2 (Buscemi et al., 2009). Fourthly, as discussed above, the iDDR domain of TRF2 prevents RNF168 and 53BP1 recruitment to telomeres, inhibiting the ATM signaling cascade downstream of ATM itself (Okamoto et al., 2013). Finally, TRF2 interacts with the $\alpha$-helix 5 domain of Ku70, preventing Ku70-Ku80 heterotetramerization and activation of NHEJ (Ribes-Zamora et al., 2013). In addition to the protective function of TRF2, POT1 protects telomeres by repressing ATR activity. POT1 binds to the ssDNA of the $3^{\prime}$-overhang and is believed to prevent the recruitment of RPA, which is crucial for ATR activation (Gong and de Lange, 2010).

Although the shelterin complex protects telomeres throughout most of the cell cycle, telomeres are briefly deprotected during and after replication, before shelterin mediated protection has been re-established on newly replicated telomeres (Verdun and Karlseder, 2006). In addition, telomeres 
appear to be in an underprotected state during mitosis when the mitotic kinase Aurora B promotes telomere deprotection (Hayashi et al., 2012). This makes mitotic telomeres vulnerable to form sister-telomere associations (Orthwein et al., 2014). Therefore, during mitosis DNA repair is actively suppressed to prevent genomic instability. This suppression is achieved by CDK1-mediated phosphorylation of RNF8 and CDK1- and PLK1-mediated phosphorylation of 53BP1 (Orthwein et al., 2014). RNF8 phosphorylation interferes with the binding of RNF8 to MDC1, thereby inhibiting its recruitment to DSBs and preventing subsequent DDR signaling. In addition, phosphorylation of 53BP1 interferes with its ability to recognize H4K20me2 and K15-ubiquitinated histone $\mathrm{H} 2 \mathrm{~A}$, which are both critical for 53BP1 recruitment to DSBs. This results in suppression of DNA repair activity during mitosis. When RNF168 and 53BP1 were artificially recruited to DSBs and telomeres, DNA repair was restored, resulting in sister telomere fusions (Orthwein et al., 2014). Thus phosphorylation of RNF168 and 53BP1 in mitosis plays an important role in maintaining genome stability and represents a shelterinindependent way of preventing DNA repair activities from acting on telomeres.

In general, it is increasingly becoming clear that ubiquitination and SUMOylation are highly involved in the regulation of the DDR triggered by DSBs in the genome. Evidence that this is also the case in response to dysfunctional telomeres has started to emerge, although there is still relatively little known about telomere-specific mechanisms. So far, the initial observations have indicated similar signaling processes in the cell's response to DSBs and dysfunctional telomeres. However, also differences between these processes have been reported. For example at telomeres, in contrast to DSBs, the Ku70-Ku80 complex is not only recruited upon damage or uncapping, but is constitutively present to prevent deletion of telomeric repeats (Wang et al., 2009). However, it also promotes NHEJ at uncapped telomeres and is therefore restricted in its activity by TRF2. TRF2, as mentioned above, inhibits Ku70$\mathrm{Ku} 80$ heterotetramerization, which interferes with $\mathrm{Ku} 70-\mathrm{Ku} 80$ activation and NHEJ induction, but does not deplete $\mathrm{Ku}$ from healthy telomeres (Ribes-Zamora et al., 2013). Furthermore, telomere deprotection has been shown to occur in different degrees, depending on the amount of TRF2 still bound to the telomeres (Cesare et al., 2013). Cells containing partially deprotected telomeres, with low amounts of TRF2 bound, will bypass the G2/M checkpoint, cycle to G1-phase and enter senescence, but will still be protected from telomeric NHEJ. However, when telomeres are completely uncapped, the DDR is fully activated and telomeres are fused through NHEJ (Cesare et al., 2013). Completely uncapped telomeres also avoid G2/M arrest, but the mechanism behind this is not yet known. These examples further emphasize that new findings regarding responses at DSBs, including ubiquitination and SUMOylation events, should also be studied at dysfunctional telomeres and vice versa, to understand whether the underlying mechanisms are identical or different between DSBs and dysfunctional telomeres.

\section{PERSPECTIVES}

A tight regulation of telomere maintenance, replication and protection is required to ensure safeguarding of genome integrity by telomeres. If factors in these processes are impaired or exhibit aberrant functions, genome stability is at risk, potentially promoting tumorigenesis. Therefore, it is crucial to further investigate the processes and factors that ensure proper telomere function. Over the last decade, the importance and functions of ubiquitination and SUMOylation at telomeres have started to become clear. These PTMs are not only essential for telomere maintenance and protection, but are also key contributors to the cell's response to dysfunctional telomeres. Although many studies have already explored ubiquitination and SUMOylation in different telomeric contexts and thereby identified various targets, the underlying mechanisms, as well as the precise contribution of PTMs are often still undetermined. Moreover, PTMs have also been shown to affect each other. So far, crosstalk between ubiquitination and SUMOylation has been shown to not only contribute to the general DDR, but also to DDR at telomeres, such as RNF4-mediated ubiquitination that was shown to require the presence of SUMO-chains (Groocock et al., 2014; Rojas-Fernandez et al., 2014). Therefore, it would be interesting to investigate whether additional crosstalk occurs at telomeres and if one aspect of telomere biology, for example DNA repair or maintenance, is more affected by the combination of ubiquitin and SUMO modifications than others. Further studies concerning telomere-specific ubiquitination and SUMOylation will be required to increase our understanding of the complex mechanisms that ensure proper telomere function or contribute to DNA repair at dysfunctional telomeres. It would be beneficial to distinguish which modifications are unique to telomeric DNA, as these might offer a tool to specifically target DNA repair at telomeres without interfering in an unwanted manner with genome-wide repair at DNA breaks.

\section{AUTHOR CONTRIBUTIONS}

All authors listed, have made substantial, direct and intellectual contribution to the work, and approved it for publication.

\section{FUNDING}

Work in the laboratory of the authors is funded by grants from the Dutch Cancer Society (NKI2012-5305), the Netherlands Organization for Scientific Research (ALW822.02.004) and the European Research Council (ERCStG 311565).

\section{ACKNOWLEDGMENTS}

We apologize to authors whose work could not be cited due to space limitations. We thank Inge de Krijger, Vera Boersma and Aurora Cerutti for critical reading of our manuscript. 


\section{REFERENCES}

Al-Wahiby, S., and Slijepcevic, P. (2005). Chromosomal aberrations involving telomeres in BRCA1 deficient human and mouse cell lines. Cytogenet. Genome. Res. 109, 491-496. doi: 10.1159/000084208

Arnoult, N., and Karlseder, J. (2015). Complex interactions between the DNAdamage response and mammalian telomeres. Nat. Struct. Mol. Biol. 22, 859-866. doi: 10.1038/nsmb.3092

Atanassov, B. S., Evrard, Y. A., Multani, A. S., Zhang, Z., Tora, L., Devys, D., et al. (2009). Gcn5 and SAGA regulate shelterin protein turnover and telomere maintenance. Mol. Cell 35, 352-364. doi: 10.1016/j.molcel.2009.06.015

Badie, S., Escandell, J. M., Bouwman, P., Carlos, A. R., Thanasoula, M., Gallardo, M. M., et al. (2010). BRCA2 acts as a RAD51 loader to facilitate telomere replication and capping. Nat. Struct. Mol. Biol. 17, 1461-1469. doi: 10.1038/ nsmb.1943

Benarroch-Popivker, D., Pisano, S., Mendez-Bermudez, A., Lototska, L., Kaur, P., Bauwens, S., et al. (2016). TRF2-mediated control of telomere DNA topology as a mechanism for chromosome-end protection. Mol. Cell 61, 274-286. doi: 10.1016/j.molcel.2015.12.009

Bhanot, M., and Smith, S. (2012). TIN2 stability is regulated by the E3 ligase Siah2. Mol. Cell. Biol. 32, 376-384. doi: 10.1128/MCB.06227-11

Boersma, V., Moatti, N., Segura-Bayona, S., Peuscher, M. H., Van Der Torre, J., Wevers, B. A., et al. (2015). MAD2L2 controls DNA repair at telomeres and DNA breaks by inhibiting 5' end resection. Nature 521, 537-540. doi: 10.1038/ nature 14216

Bothmer, A., Robbiani, D. F., Feldhahn, N., Gazumyan, A., Nussenzweig, A., and Nussenzweig, M. C. (2010). 53BP1 regulates DNA resection and the choice between classical and alternative end joining during class switch recombination. J. Exp. Med. 207, 855-865. doi: 10.1084/jem.20100244

Brown, J. S., and Jackson, S. P. (2015). Ubiquitylation, neddylation and the DNA damage response. Open Biol. 5:150018. doi: 10.1098/rsob.150018

Buscemi, G., Zannini, L., Fontanella, E., Lecis, D., Lisanti, S., and Delia, D. (2009). The shelterin protein TRF2 inhibits Chk2 activity at telomeres in the absence of DNA damage. Curr. Biol. 19, 874-879. doi: 10.1016/j.cub.2009.03.064

Callen, E., Di Virgilio, M., Kruhlak, M. J., Nieto-Soler, M., Wong, N., Chen, H. T., et al. (2013). 53BP1 mediates productive and mutagenic DNA repair through distinct phosphoprotein interactions. Cell 153, 1266-1280. doi: 10.1016/j.cell. 2013.05.023

Cesare, A. J., Hayashi, M. T., Crabbe, L., and Karlseder, J. (2013). The telomere deprotection response is functionally distinct from the genomic DNA damage response. Mol. Cell 51, 141-155. doi: 10.1016/j.molcel.2013.06.006

Chapman, J. R., Barral, P., Vannier, J. B., Borel, V., Steger, M., Tomas-Loba, A., et al. (2013). RIF1 is essential for 53BP1-dependent nonhomologous end joining and suppression of DNA double-strand break resection. Mol. Cell 49, 858-871. doi: 10.1016/j.molcel.2013.01.002

Chen, L. Y., and Lingner, J. (2013). CST for the grand finale of telomere replication. Nucleus 4, 277-282. doi: 10.4161/nucl.25701

Chow, T. T., Zhao, Y., Mak, S. S., Shay, J. W., and Wright, W. E. (2012). Early and late steps in telomere overhang processing in normal human cells: the position of the final RNA primer drives telomere shortening. Genes Dev. 26, 1167-1178. doi: $10.1101 /$ gad.187211.112

Churikov, D., Charifi, F., Eckert-Boulet, N., Silva, S., Simon, M. N., Lisby, M., et al. (2016). SUMO-dependent relocalization of eroded telomeres to nuclear pore complexes controls telomere recombination. Cell Rep. 15, 1242-1253. doi: 10.1016/j.celrep.2016.04.008

Ciechanover, A., Elias, S., Heller, H., and Hershko, A. (1982). "Covalent affinity" purification of ubiquitin-activating enzyme. J. Biol. Chem. 257, 2537-2542.

Cimprich, K. A., and Cortez, D. (2008). ATR: an essential regulator of genome integrity. Nat. Rev. Mol. Cell Biol. 9, 616-627. doi: 10.1038/nrm2450

Citterio, E. (2015). Fine-tuning the ubiquitin code at DNA double-strand breaks: deubiquitinating enzymes at work. Front. Genet. 6:282. doi: 10.3389/fgene.2015. 00282

Coleman, K. A., and Greenberg, R. A. (2011). The BRCA1-RAP80 complex regulates DNA repair mechanism utilization by restricting end resection. J. Biol. Chem. 286, 13669-13680. doi: 10.1074/jbc.M110.213728

Crabbe, L., Verdun, R. E., Haggblom, C. I., and Karlseder, J. (2004). Defective telomere lagging strand synthesis in cells lacking WRN helicase activity. Science 306, 1951-1953. doi: 10.1126/science.1103619
Cubenas-Potts, C., and Matunis, M. J. (2013). SUMO: a multifaceted modifier of chromatin structure and function. Dev. Cell 24, 1-12. doi: 10.1016/j.devcel. 2012.11.020

d'Adda di Fagagna, F., Reaper, P. M., Clay-Farrace, L., Fiegler, H., Carr, P., Von Zglinicki, T., et al. (2003). A DNA damage checkpoint response in telomereinitiated senescence. Nature 426, 194-198. doi: 10.1038/nature02118

Davoli, T., and de Lange, T. (2011). The causes and consequences of polyploidy in normal development and cancer. Annu. Rev. Cell Dev. Biol. 27, 585-610. doi: 10.1146/annurev-cellbio-092910-154234

Denchi, E. L., and de Lange, T. (2007). Protection of telomeres through independent control of ATM and ATR by TRF2 and POT1. Nature 448, 1068-1071. doi: 10.1038/nature06065

Di Virgilio, M., Callen, E., Yamane, A., Zhang, W., Jankovic, M., Gitlin, A. D., et al. (2013). Rif1 prevents resection of DNA breaks and promotes immunoglobulin class switching. Science 339, 711-715. doi: 10.1126/science.1230624

Dimitrova, N., Chen, Y. C., Spector, D. L., and De Lange, T. (2008). 53BP1 promotes non-homologous end joining of telomeres by increasing chromatin mobility. Nature 456, 524-528. doi: 10.1038/nature07433

Dimitrova, N., and de Lange, T. (2006). MDC1 accelerates nonhomologous endjoining of dysfunctional telomeres. Genes Dev. 20, 3238-3243. doi: 10.1101/gad. 1496606

Doksani, Y., and de Lange, T. (2014). The role of double-strand break repair pathways at functional and dysfunctional telomeres. Cold Spring Harb. Perspect. Biol. 6:a016576. doi: 10.1101/cshperspect.a016576

Doksani, Y., Wu, J. Y., De Lange, T., and Zhuang, X. (2013). Super-resolution fluorescence imaging of telomeres reveals TRF2-dependent T-loop formation. Cell 155, 345-356. doi: 10.1016/j.cell.2013.09.048

Edwards, D. N., Orren, D. K., and Machwe, A. (2014). Strand exchange of telomeric DNA catalyzed by the Werner syndrome protein (WRN) is specifically stimulated by TRF2. Nucleic Acids Res. 42, 7748-7761. doi: 10.1093/nar/ gku454

Escribano-Diaz, C., Orthwein, A., Fradet-Turcotte, A., Xing, M., Young, J. T., Tkac, J., et al. (2013). A cell cycle-dependent regulatory circuit composed of 53BP1-RIF1 and BRCA1-CtIP controls DNA repair pathway choice. Mol. Cell 49, 872-883. doi: 10.1016/j.molcel.2013.01.001

Feng, L., Fong, K. W., Wang, J., Wang, W., and Chen, J. (2013). RIF1 counteracts BRCA1-mediated end resection during DNA repair. J. Biol. Chem. 288, 11135-11143. doi: 10.1074/jbc.M113.457440

Fradet-Turcotte, A., Canny, M. D., Escribano-Diaz, C., Orthwein, A., Leung, C. C., Huang, H., et al. (2013). 53BP1 is a reader of the DNA-damage-induced H2A Lys 15 ubiquitin mark. Nature 499, 50-54. doi: 10.1038/nature12318

Fujita, K., Horikawa, I., Mondal, A. M., Jenkins, L. M., Appella, E., Vojtesek, B., et al. (2010). Positive feedback between p53 and TRF2 during telomere-damage signalling and cellular senescence. Nat. Cell Biol. 12, 1205-1212. doi: 10.1038/ ncb2123

Gao, X., Wang, W., Yang, H., Wu, L., He, Z., Zhou, S., et al. (2016). UBE2D3 gene overexpression increases radiosensitivity of EC109 esophageal cancer cells in vitro and in vivo. Oncotarget 7, 32543-32553. doi: 10.18632/oncotarget. 8869

Garg, M., Gurung, R. L., Mansoubi, S., Ahmed, J. O., Dave, A., Watts, F. Z., et al. (2014). Tpz1TPP1 SUMOylation reveals evolutionary conservation of SUMOdependent Stn1 telomere association. EMBO Rep. 15, 871-877. doi: 10.15252/ embr.201438919

Geiss-Friedlander, R., and Melchior, F. (2007). Concepts in sumoylation: a decade on. Nat. Rev. Mol. Cell Biol. 8, 947-956. doi: 10.1038/nrm2293

Gong, Y., and de Lange, T. (2010). A Shld1-controlled POT1a provides support for repression of ATR signaling at telomeres through RPA exclusion. Mol. Cell 40, 377-387. doi: 10.1016/j.molcel.2010.10.016

Greider, C. W., and Blackburn, E. H. (1985). Identification of a specific telomere terminal transferase activity in Tetrahymena extracts. Cell 43, 405-413.

Griffith, J. D., Comeau, L., Rosenfield, S., Stansel, R. M., Bianchi, A., Moss, H., et al. (1999). Mammalian telomeres end in a large duplex loop. Cell 97, 503-514.

Groocock, L. M., Nie, M., Prudden, J., Moiani, D., Wang, T., Cheltsov, A., et al. (2014). RNF4 interacts with both SUMO and nucleosomes to promote the DNA damage response. EMBO Rep. 15, 601-608. doi: 10.1002/embr.201338369

Gudjonsson, T., Altmeyer, M., Savic, V., Toledo, L., Dinant, C., Grofte, M., et al. (2012). TRIP12 and UBR5 suppress spreading of chromatin ubiquitylation at damaged chromosomes. Cell 150, 697-709. doi: 10.1016/j.cell.2012.06.039 
Ha, G. H., Kim, H. S., Go, H., Lee, H., Seimiya, H., Chung, D. H., et al. (2012). Tankyrase-1 function at telomeres and during mitosis is regulated by Polo-like kinase-1-mediated phosphorylation. Cell Death Differ. 19, 321-332. doi: $10.1038 /$ cdd. 2011.101

Hang, L. E., Liu, X., Cheung, I., Yang, Y., and Zhao, X. (2011). SUMOylation regulates telomere length homeostasis by targeting Cdc13. Nat. Struct. Mol. Biol. 18, 920-926. doi: 10.1038/nsmb.2100

Harley, C. B., Futcher, A. B., and Greider, C. W. (1990). Telomeres shorten during ageing of human fibroblasts. Nature 345, 458-460. doi: 10.1038/345458a0

Hayashi, M. T., Cesare, A. J., Fitzpatrick, J. A., Lazzerini-Denchi, E., and Karlseder, J. (2012). A telomere-dependent DNA damage checkpoint induced by prolonged mitotic arrest. Nat. Struct. Mol. Biol. 19, 387-394. doi: 10.1038/ nsmb. 2245

Her, J., Jeong, Y. Y., and Chung, I. K. (2015). PIAS1-mediated sumoylation promotes STUbL-dependent proteasomal degradation of the human telomeric protein TRF2. FEBS Lett. 589, 3277-3286. doi: 10.1016/j.febslet.2015.09.030

Her, Y. R., and Chung, I. K. (2009). Ubiquitin Ligase RLIM modulates telomere length homeostasis through a Proteolysis of TRF1. J. Biol. Chem. 284, 8557-8566. doi: 10.1074/jbc.M806702200

Hershko, A., Heller, H., Elias, S., and Ciechanover, A. (1983). Components of ubiquitin-protein ligase system. Resolution, affinity purification, and role in protein breakdown. J. Biol. Chem. 258, 8206-8214.

Hirai, Y., Tamura, M., Otani, J., and Ishikawa, F. (2016). NEK6-mediated phosphorylation of human TPP1 regulates telomere length through telomerase recruitment. Genes Cells 21, 874-889. doi: 10.1111/gtc.12391

Holtrich, U., Wolf, G., Brauninger, A., Karn, T., Bohme, B., RubsamenWaigmann, H., et al. (1994). Induction and down-regulation of PLK, a human serine/threonine kinase expressed in proliferating cells and tumors. Proc. Natl. Acad. Sci. U.S.A. 91, 1736-1740.

Huang, C., Dai, X., and Chai, W. (2012). Human Stn1 protects telomere integrity by promoting efficient lagging-strand synthesis at telomeres and mediating C-strand fill-in. Cell Res. 22, 1681-1695. doi: 10.1038/cr.2012.132

Huang, Y., Sun, L., Liu, N., Wei, Q., Jiang, L., Tong, X., et al. (2015). Polo-like Kinase 1 (Plk1) Up-regulates telomerase activity by affecting human telomerase reverse transcriptase (hTERT) stability. J. Biol. Chem. 290, 18865-18873. doi: $10.1074 /$ jbc.M114.635375

Jacobs, J. J. (2013). Loss of telomere protection: consequences and opportunities. Front. Oncol. 3:88. doi: 10.3389/fonc.2013.00088

Jacobs, J. J., and de Lange, T. (2005). p16INK4a as a second effector of the telomere damage pathway. Cell Cycle 4, 1364-1368. doi: 10.4161/cc.4.10.2104

Johnson, E. S. (2004). Protein modification by SUMO. Annu. Rev. Biochem. 73, 355-382. doi: 10.1146/annurev.biochem.73.011303.074118

Kabir, S., Hockemeyer, D., and De Lange, T. (2014). TALEN gene knockouts reveal no requirement for the conserved human shelterin protein Rap1 in telomere protection and length regulation. Cell Rep. 9, 1273-1280. doi: 10.1016/j.celrep. 2014.10.014

Karlseder, J., Hoke, K., Mirzoeva, O. K., Bakkenist, C., Kastan, M. B., Petrini, J. H., et al. (2004). The telomeric protein TRF2 binds the ATM kinase and can inhibit the ATM-dependent DNA damage response. PLoS Biol. 2:E240. doi: 10.1371 /journal.pbio.0020240

Kerscher, O. (2007). SUMO junction-what's your function? New insights through SUMO-interacting motifs. EMBO Rep. 8, 550-555. doi: 10.1038/sj.embor. 7400980

Kim, J., and Chung, I. K. (2014). The splicing factor U2AF65 stabilizes TRF1 protein by inhibiting its ubiquitin-dependent proteolysis. Biochem. Biophys. Res. Commun. 443, 1124-1130. doi: 10.1016/j.bbrc.2013.12.118

Kim, J. H., Park, S. M., Kang, M. R., Oh, S. Y., Lee, T. H., Muller, M. T., et al. (2005). Ubiquitin ligase MKRN1 modulates telomere length homeostasis through a proteolysis of hTERT. Genes Dev. 19, 776-781. doi: 10.1101/gad.128 9405

Kolas, N. K., Chapman, J. R., Nakada, S., Ylanko, J., Chahwan, R., Sweeney, F. D., et al. (2007). Orchestration of the DNA-damage response by the RNF8 ubiquitin ligase. Science 318, 1637-1640. doi: 10.1126/science.1150034

Komander, D., Clague, M. J., and Urbe, S. (2009). Breaking the chains: structure and function of the deubiquitinases. Nat. Rev. Mol. Cell Biol. 10, 550-563. doi: $10.1038 / \mathrm{nrm} 2731$

Komander, D., and Rape, M. (2012). The ubiquitin code. Annu. Rev. Biochem. 81, 203-229. doi: 10.1146/annurev-biochem-060310-170328
Lallemand-Breitenbach, V., Jeanne, M., Benhenda, S., Nasr, R., Lei, M., Peres, L., et al. (2008). Arsenic degrades PML or PML-RARalpha through a SUMOtriggered RNF4/ubiquitin-mediated pathway. Nat. Cell Biol. 10, 547-555. doi: $10.1038 /$ ncb 1717

Lazzerini-Denchi, E., and Sfeir, A. (2016). Stop pulling my strings - what telomeres taught us about the DNA damage response. Nat. Rev. Mol. Cell Biol. 17, 364-378. doi: 10.1038/nrm.2016.43

Lee, J. H., Khadka, P., Baek, S. H., and Chung, I. K. (2010). CHIP promotes human telomerase reverse transcriptase degradation and negatively regulates telomerase activity. J. Biol. Chem. 285, 42033-42045. doi: 10.1074/jbc.M110. 149831

Lee, T. H., Perrem, K., Harper, J. W., Lu, K. P., and Zhou, X. Z. (2006). The F-box protein FBX4 targets PIN2/TRF1 for ubiquitin-mediated degradation and regulates telomere maintenance. J. Biol. Chem. 281, 759-768. doi: 10.1074/ jbc.M509855200

Lescasse, R., Pobiega, S., Callebaut, I., and Marcand, S. (2013). End-joining inhibition at telomeres requires the translocase and polySUMO-dependent ubiquitin ligase Uls1. EMBO J. 32, 805-815. doi: 10.1038/emboj.2013.24

Liu, D., Safari, A., O'connor, M. S., Chan, D. W., Laegeler, A., Qin, J., et al. (2004). PTOP interacts with POT1 and regulates its localization to telomeres. Nat. Cell Biol. 6, 673-680. doi: 10.1038/ncb1142

Loayza, D., and De Lange, T. (2003). POT1 as a terminal transducer of TRF1 telomere length control. Nature 423, 1013-1018. doi: 10.1038/nature01688

Mailand, N., Bekker-Jensen, S., Faustrup, H., Melander, F., Bartek, J., Lukas, C., et al. (2007). RNF8 ubiquitylates histones at DNA double-strand breaks and promotes assembly of repair proteins. Cell 131, 887-900. doi: 10.1016/j.cell. 2007.09.040

Manis, J. P., Morales, J. C., Xia, Z., Kutok, J. L., Alt, F. W., and Carpenter, P. B. (2004). 53BP1 links DNA damage-response pathways to immunoglobulin heavy chain class-switch recombination. Nat. Immunol. 5, 481-487. doi: 10.1038/ ni1067

Martinez, P., and Blasco, M. A. (2015). Replicating through telomeres: a means to an end. Trends Biochem. Sci. 40, 504-515. doi: 10.1016/j.tibs.2015.06.003

Martinez, P., Gomez-Lopez, G., Pisano, D. G., Flores, J. M., and Blasco, M. A. (2016). A genetic interaction between RAP1 and telomerase reveals an unanticipated role for RAP1 in telomere maintenance. Aging Cell doi: 10.1111/ acel.12517 [Epub ahead of print].

Mattiroli, F., Vissers, J. H., Van Dijk, W. J., Ikpa, P., Citterio, E., Vermeulen, W., et al. (2012). RNF168 ubiquitinates K13-15 on H2A/H2AX to drive DNA damage signaling. Cell 150, 1182-1195. doi: 10.1016/j.cell.2012.08.005

Miyagawa, K., Low, R. S., Santosa, V., Tsuji, H., Moser, B. A., Fujisawa, S., et al. (2014). SUMOylation regulates telomere length by targeting the shelterin subunit Tpz1(Tpp1) to modulate shelterin-Stn1 interaction in fission yeast. Proc. Natl. Acad. Sci. U.S.A. 111, 5950-5955. doi: 10.1073/pnas.1401359111

Miyoshi, T., Kanoh, J., Saito, M., and Ishikawa, F. (2008). Fission yeast Pot1Tpp1 protects telomeres and regulates telomere length. Science 320, 1341-1344. doi: 10.1126/science.1154819

Mukhopadhyay, D., and Dasso, M. (2007). Modification in reverse: the SUMO proteases. Trends Biochem. Sci. 32, 286-295. doi: 10.1016/j.tibs.2007.05.002

Nagai, S., Dubrana, K., Tsai-Pflugfelder, M., Davidson, M. B., Roberts, T. M., Brown, G. W., et al. (2008). Functional targeting of DNA damage to a nuclear pore-associated SUMO-dependent ubiquitin ligase. Science 322, 597-602. doi: $10.1126 /$ science. 1162790

Nandakumar, J., and Cech, T. R. (2013). Finding the end: recruitment of telomerase to telomeres. Nat. Rev. Mol. Cell Biol. 14, 69-82. doi: 10.1038/nrm3505

O'Connor, M. S., Safari, A., Xin, H., Liu, D., and Songyang, Z. (2006). A critical role for TPP1 and TIN2 interaction in high-order telomeric complex assembly. Proc. Natl. Acad. Sci. U.S.A. 103, 11874-11879. doi: 10.1073/pnas.0605303103

Oh, W., Lee, E. W., Lee, D., Yang, M. R., Ko, A., Yoon, C. H., et al. (2010). Hdm2 negatively regulates telomerase activity by functioning as an E3 ligase of hTERT. Oncogene 29, 4101-4112. doi: 10.1038/onc.2010.160

Okamoto, K., Bartocci, C., Ouzounov, I., Diedrich, J. K., Yates, J. R. III, and Denchi, E. L. (2013). A two-step mechanism for TRF2-mediated chromosome-end protection. Nature 494, 502-505. doi: 10.1038/nature11873

Orthwein, A., Fradet-Turcotte, A., Noordermeer, S. M., Canny, M. D., Brun, C. M., Strecker, J., et al. (2014). Mitosis inhibits DNA double-strand break repair to guard against telomere fusions. Science 344, 189-193. doi: 10.1126/science. 1248024 
Ouyang, J., Garner, E., Hallet, A., Nguyen, H. D., Rickman, K. A., Gill, G., et al. (2015). Noncovalent interactions with SUMO and ubiquitin orchestrate distinct functions of the SLX4 complex in genome maintenance. Mol. Cell 57, 108-122. doi: 10.1016/j.molcel.2014.11.015

Palm, W., and de Lange, T. (2008). How shelterin protects mammalian telomeres. Annu. Rev. Genet. 42, 301-334. doi: 10.1146/annurev.genet.41.110306.130350

Pellegrini, L. (2012). The Pol alpha-primase complex. Subcell. Biochem. 62, 157-169. doi: 10.1007/978-94-007-4572-8_9

Peuscher, M. H., and Jacobs, J. J. (2011). DNA-damage response and repair activities at uncapped telomeres depend on RNF8. Nat. Cell Biol. 13, 1139-1145. doi: $10.1038 /$ ncb2326

Peuscher, M. H., and Jacobs, J. J. (2012). Posttranslational control of telomere maintenance and the telomere damage response. Cell Cycle 11, 1524-1534. doi: $10.4161 /$ cc. 19847

Pickett, H. A., and Reddel, R. R. (2015). Molecular mechanisms of activity and derepression of alternative lengthening of telomeres. Nat. Struct. Mol. Biol. 22, 875-880. doi: 10.1038/nsmb.3106

Potts, P. R., and Yu, H. (2007). The SMC5/6 complex maintains telomere length in ALT cancer cells through SUMOylation of telomere-binding proteins. Nat. Struct. Mol. Biol. 14, 581-590. doi: 10.1038/nsmb1259

Price, C. M., Boltz, K. A., Chaiken, M. F., Stewart, J. A., Beilstein, M. A., and Shippen, D. E. (2010). Evolution of CST function in telomere maintenance. Cell Cycle 9, 3157-3165. doi: 10.4161/cc.9.16.12547

Rai, R., Chen, Y., Lei, M., and Chang, S. (2016). TRF2-RAP1 is required to protect telomeres from engaging in homologous recombination-mediated deletions and fusions. Nat. Commun. 7:10881. doi: 10.1038/ncomms10881

Rai, R., Li, J. M., Zheng, H., Lok, G. T., Deng, Y., Huen, M. S., et al. (2011). The E3 ubiquitin ligase Rnf8 stabilizes Tpp1 to promote telomere end protection. Nat. Struct. Mol. Biol. 18, 1400-1407. doi: 10.1038/nsmb.2172

Reveal, P. M., Henkels, K. M., and Turchi, J. J. (1997). Synthesis of the mammalian telomere lagging strand in vitro. J. Biol. Chem. 272, 11678-11681.

Ribes-Zamora, A., Indiviglio, S. M., Mihalek, I., Williams, C. L., and Bertuch, A. A. (2013). TRF2 interaction with Ku heterotetramerization interface gives insight into c-NHEJ prevention at human telomeres. Cell Rep. 5, 194-206. doi: 10.1016/j.celrep.2013.08.040

Rivera, T., Haggblom, C., Cosconati, S., and Karlseder, J. (2017). A balance between elongation and trimming regulates telomere stability in stem cells. Nat. Struct. Mol. Biol. 24, 30-39. doi: 10.1038/nsmb.3335

Rojas-Fernandez, A., Plechanovova, A., Hattersley, N., Jaffray, E., Tatham, M. H., and Hay, R. T. (2014). SUMO chain-induced dimerization activates RNF4. Mol. Cell 53, 880-892. doi: 10.1016/j.molcel.2014.02.031

Salvatico, J., Kim, J. H., Chung, I. K., and Muller, M. T. (2010). Differentiation linked regulation of telomerase activity by Makorin-1. Mol. Cell. Biochem. 342, 241-250. doi: 10.1007/s11010-010-0490-x

Sarthy, J., Bae, N. S., Scrafford, J., and Baumann, P. (2009). Human RAP1 inhibits non-homologous end joining at telomeres. EMBO J. 28, 3390-3399. doi: 10. 1038/emboj.2009.275

Saville, M. K., Sparks, A., Xirodimas, D. P., Wardrop, J., Stevenson, L. F., Bourdon, J. C., et al. (2004). Regulation of p53 by the ubiquitin-conjugating enzymes UbcH5B/C in vivo. J. Biol. Chem. 279, 42169-42181. doi: 10.1074/jbc. M403362200

Sfeir, A., Kabir, S., Van Overbeek, M., Celli, G. B., and De Lange, T. (2010). Loss of Rap1 induces telomere recombination in the absence of NHEJ or a DNA damage signal. Science 327, 1657-1661. doi: 10.1126/science. 1185100

Sfeir, A., Kosiyatrakul, S. T., Hockemeyer, D., Macrae, S. L., Karlseder, J., Schildkraut, C. L., et al. (2009). Mammalian telomeres resemble fragile sites and require TRF1 for efficient replication. Cell 138, 90-103. doi: 10.1016/j.cell.2009. 06.021

Shao, G., Lilli, D. R., Patterson-Fortin, J., Coleman, K. A., Morrissey, D. E., and Greenberg, R. A. (2009). The Rap80-BRCC36 de-ubiquitinating enzyme complex antagonizes RNF8-Ubc13-dependent ubiquitination events at DNA double strand breaks. Proc. Natl. Acad. Sci. U.S.A. 106, 3166-3171. doi: 10.1073/ pnas.0807485106

Smith, S., Giriat, I., Schmitt, A., and De Lange, T. (1998). Tankyrase, a poly(ADPribose) polymerase at human telomeres. Science 282, 1484-1487.

Stansel, R. M., De Lange, T., and Griffith, J. D. (2001). T-loop assembly in vitro involves binding of TRF2 near the 3' telomeric overhang. EMBO J. 20, 5532-5540. doi: 10.1093/emboj/20.19.5532
Stewart, J. A., Chaiken, M. F., Wang, F., and Price, C. M. (2012). Maintaining the end: roles of telomere proteins in end-protection, telomere replication and length regulation. Mutat. Res. 730, 12-19. doi: 10.1016/j.mrfmmm.2011.08.011

Tacconi, E. M., and Tarsounas, M. (2015). How homologous recombination maintains telomere integrity. Chromosoma 124, 119-130. doi: 10.1007/s00412014-0497-2

Tatham, M. H., Geoffroy, M. C., Shen, L., Plechanovova, A., Hattersley, N., Jaffray, E. G., et al. (2008). RNF4 is a poly-SUMO-specific E3 ubiquitin ligase required for arsenic-induced PML degradation. Nat. Cell Biol. 10, 538-546. doi: 10.1038/ ncb 1716

Thorslund, T., Ripplinger, A., Hoffmann, S., Wild, T., Uckelmann, M., Villumsen, B., et al. (2015). Histone H1 couples initiation and amplification of ubiquitin signalling after DNA damage. Nature 527, 389-393. doi: 10.1038/ nature 15401

Uringa, E. J., Lisaingo, K., Pickett, H. A., Brind'amour, J., Rohde, J. H., Zelensky, A., et al. (2012). RTEL1 contributes to DNA replication and repair and telomere maintenance. Mol. Biol. Cell 23, 2782-2792. doi: 10.1091/mbc.E12-030179

Vannier, J. B., Pavicic-Kaltenbrunner, V., Petalcorin, M. I., Ding, H., and Boulton, S. J. (2012). RTEL1 dismantles T loops and counteracts telomeric G4-DNA to maintain telomere integrity. Cell 149, 795-806. doi: 10.1016/j.cell.2012. 03.030

Vannier, J. B., Sandhu, S., Petalcorin, M. I., Wu, X., Nabi, Z., Ding, H., et al. (2013). RTEL1 is a replisome-associated helicase that promotes telomere and genome-wide replication. Science 342, 239-242. doi: 10.1126/science.1241779

Verdun, R. E., and Karlseder, J. (2006). The DNA damage machinery and homologous recombination pathway act consecutively to protect human telomeres. Cell 127, 709-720. doi: 10.1016/j.cell.2006.09.034

Wang, C., Xiao, H., Ma, J., Zhu, Y., Yu, J., Sun, L., et al. (2013). The F-box protein beta-TrCP promotes ubiquitination of TRF1 and regulates the ALT-associated PML bodies formation in U2OS cells. Biochem. Biophys. Res. Commun. 434, 728-734. doi: 10.1016/j.bbrc.2013.03.096

Wang, C., Yu, J., Yuan, K., Lan, J., Jin, C., and Huang, H. (2010). Plk1-mediated mitotic phosphorylation of PinX1 regulates its stability. Eur. J. Cell Biol. 89, 748-756. doi: 10.1016/j.ejcb.2010.05.005

Wang, R. C., Smogorzewska, A., and De Lange, T. (2004). Homologous recombination generates T-loop-sized deletions at human telomeres. Cell 119, 355-368. doi: 10.1016/j.cell.2004.10.011

Wang, W., Yang, L., Hu, L., Li, F., Ren, L., Yu, H., et al. (2013). Inhibition of UBE2D3 expression attenuates radiosensitivity of MCF-7 human breast cancer cells by increasing hTERT expression and activity. PLoS ONE 8:e64660. doi: 10.1371/journal.pone.0064660

Wang, Y., Ghosh, G., and Hendrickson, E. A. (2009). Ku86 represses lethal telomere deletion events in human somatic cells. Proc. Natl. Acad. Sci. U.S.A. 106, 12430-12435. doi: 10.1073/pnas.0903362106

Wang, Z., Zhang, H., Liu, J., Cheruiyot, A., Lee, J. H., Ordog, T., et al. (2016). USP51 deubiquitylates $\mathrm{H} 2 \mathrm{AK} 13,15 \mathrm{ub}$ and regulates DNA damage response. Genes Dev. 30, 946-959. doi: 10.1101/gad.271841.115

Weisshaar, S. R., Keusekotten, K., Krause, A., Horst, C., Springer, H. M., Gottsche, K., et al. (2008). Arsenic trioxide stimulates SUMO-2/3 modification leading to RNF4-dependent proteolytic targeting of PML. FEBS Lett. 582, 3174-3178. doi: 10.1016/j.febslet.2008.08.008

Wilson, J. S., Tejera, A. M., Castor, D., Toth, R., Blasco, M. A., and Rouse, J. (2013). Localization-dependent and -independent roles of SLX4 in regulating telomeres. Cell Rep. 4, 853-860. doi: 10.1016/j.celrep.2013.07.033

Wu, P., Van Overbeek, M., Rooney, S., and De Lange, T. (2010). Apollo contributes to $\mathrm{G}$ overhang maintenance and protects leading-end telomeres. Mol. Cell 39, 606-617. doi: 10.1016/j.molcel.2010.06.031

Xu, G., Chapman, J. R., Brandsma, I., Yuan, J., Mistrik, M., Bouwman, P., et al. (2015). REV7 counteracts DNA double-strand break resection and affects PARP inhibition. Nature 521, 541-544. doi: 10.1038/nature14328

Yang, H., Wu, L., Ke, S., Wang, W., Yang, L., Gao, X., et al. (2016). Downregulation of ubiquitin-conjugating enzyme UBE2D3 promotes telomere maintenance and radioresistance of Eca-109 human esophageal carcinoma cells. J. Cancer 7, 1152-1162. doi: 10.7150/jca.14745

Ye, J. Z., and de Lange, T. (2004). TIN2 is a tankyrase 1 PARP modulator in the TRF1 telomere length control complex. Nat. Genet. 36, 618-623. doi: 10.1038/ ng1360 
Yeager, T. R., Neumann, A. A., Englezou, A., Huschtscha, L. I., Noble, J. R., and Reddel, R. R. (1999). Telomerase-negative immortalized human cells contain a novel type of promyelocytic leukemia (PML) body. Cancer Res. 59, 4175-4179.

Yoo, J. E., Oh, B. K., and Park, Y. N. (2009). Human PinX1 mediates TRF1 accumulation in nucleolus and enhances TRF1 binding to telomeres. J. Mol. Biol. 388, 928-940. doi: 10.1016/j.jmb.2009.02.051

Zemp, I., and Lingner, J. (2014). The shelterin component TPP1 is a binding partner and substrate for the deubiquitinating enzyme USP7. J. Biol. Chem. 289, 28595-28606. doi: 10.1074/jbc.M114.596056

Zeng, Z., Wang, W., Yang, Y., Chen, Y., Yang, X., Diehl, J. A., et al. (2010). Structural basis of selective ubiquitination of TRF1 by SCFFbx4. Dev. Cell 18, 214-225. doi: 10.1016/j.devcel.2010.01.007

Zhong, F. L., Batista, L. F., Freund, A., Pech, M. F., Venteicher, A. S., and Artandi, S. E. (2012). TPP1 OB-fold domain controls telomere maintenance by recruiting telomerase to chromosome ends. Cell 150, 481-494. doi: 10.1016/j. cell.2012.07.012

Zhou, X. Z., and Lu, K. P. (2001). The Pin2/TRF1-interacting protein PinX1 is a potent telomerase inhibitor. Cell 107, 347-359.
Zimmer, J., Tacconi, E. M., Folio, C., Badie, S., Porru, M., Klare, K., et al. (2016). Targeting BRCA1 and BRCA2 Deficiencies with G-QuadruplexInteracting Compounds. Mol. Cell 61, 449-460. doi: 10.1016/j.molcel.2015. 12.004

Zimmermann, M., Lottersberger, F., Buonomo, S. B., Sfeir, A., and De Lange, T. (2013). 53BP1 regulates DSB repair using Rif1 to control 5' end resection. Science 339, 700-704. doi: 10.1126/science.1231573

Conflict of Interest Statement: The authors declare that the research was conducted in the absence of any commercial or financial relationships that could be construed as a potential conflict of interest.

Copyright (c) 2017 Yalçin, Selenz and Jacobs. This is an open-access article distributed under the terms of the Creative Commons Attribution License (CC BY). The use, distribution or reproduction in other forums is permitted, provided the original author(s) or licensor are credited and that the original publication in this journal is cited, in accordance with accepted academic practice. No use, distribution or reproduction is permitted which does not comply with these terms. 